\title{
The Emerging Roles of Non-Coding RNAs in Cataract
}

\author{
Jie Li' ${ }^{1}$, Lei Ge' ${ }^{1}$ Xiaoqin Wang' ${ }^{2}$ Zhaowu $\mathrm{Ma}^{3^{*}}$ \\ ${ }^{1}$ Ophthalmology, Yangtze University Health Science Center, Jingzhou, China \\ ${ }^{2}$ Department of Ophthalmology, Jingzhou Central Hospital, Jingzhou, China \\ ${ }^{3}$ School of Basic Medicine, Health Science Center, Yangtze University, Jingzhou, China \\ Email: *zhaowu823@126.com
}

How to cite this paper: Li, J., Ge, L., Wang, X.Q. and Ma, Z.W. (2020) The Emerging Roles of Non-Coding RNAs in Cataract. Yangtze Medicine, 4, 218-228. https://doi.org/10.4236/ym.2020.43021

Received: September 16, 2019

Accepted: June 27, 2020

Published: June 30, 2020

Copyright (c) 2020 by author(s) and Scientific Research Publishing Inc. This work is licensed under the Creative Commons Attribution International License (CC BY 4.0).

http://creativecommons.org/licenses/by/4.0/ (c) (i) Open Access

\begin{abstract}
Non-coding RNAs (ncRNAs) are a large class of transcripts lacking evident protein coding potential, and play versatile roles in a diverse range of physiological and pathological processes. Mounting evidences have indicated that ncRNAs are aberrantly expressed in a wealth of diseases such as cataract. Cataract is a cloudy lens caused by radiation, age, drugs and other factors. NcRNAs, including microRNAs, long non-coding RNAs, circular RNAs, have been identified to regulate the occurrence and development of cataract. Current studies indicate that ncRNAs exert the multifaceted functions in the lens of cataract patients and have been proved as potential diagnostic biomarkers or therapeutic targets for cataracts. This review summarizes the study of relationship between the lens and ncRNAs, which can provide a novel insight into the pathogenesis of cataract.
\end{abstract}

\section{Keywords}

Cataract, PCO, microRNAs, Long Non-Coding RNAs, Circular RNAs

\section{Introduction}

In the coming decades, cataract and cataract blindness will continue being a leading public health issue in China due to the aging population [1]. Cataract is one of the main causes of blindness around the world. Many factors can increase the incidence of cataracts, such as inheritance, aging, radiation, injury, diabetes mellitus and medication [2] [3] [4]. Lens Opacification Classification System III (LOCS) is the most commonly used for evaluating. Cataract is divided into cortical cataract, nuclear cataract and posterior sub-capsular cataract. Phacoemulsification is a satisfactory method for the treatment of cataract, but there are also 
many postoperative complications [5] [6] [7]. Posterior capsular opacification (PCO) is the most common complication after a few years of surgery [8]. Therefore, the prevention and treatment of cataract has become particularly important. It is necessary to study the mechanism of age-related cataract (ARC), diabetic cataract (DC) and PCO objective to explore a new therapeutic target for cataract.

In the entire human genome, about 70 percent of the DNA are transcribed into RNA, but only 2 percent can be used to encode proteins. Although non-coding RNA does not encode a protein, such RNAs do contain information and function. These non-coding RNAs mainly include microRNAs, tsRNA (tRNA-derived small RNA), circular RNAs, long coding RNAs and pseudogene [9]. The main function of non-coding RNA include: to participate in the stability of mRNA and the regulation of translation level; to participate in the transport of protein; to participate in the procession and modification of RNA and to affect the structure of chromosomes [10]. Most of those definite functions are still unknown in cataract, so non-coding RNAs have been the focus of researchers. Researches have shown that the abnormal expression of non-coding RNAs (miRNA, lncRNA, circRNA) can lead to the disorder of normal lens development, apoptosis of lens epithelial cells, the disorder of fiber cells and the transparency of the lens decreases, which leads to the mechanism of cataract [11] [12] [13].

In this review, we summarize the deregulations and discuss the pathological implications of miRNAs, IncRNAs and circRNAs in ophthalmology. We highlight the biological functions of non-coding RNAs and their targets, pathways in ARC, DC or PCO. Finally, we discuss the potential roles of ncRNAs as diagnostic tools and therapeutic targets in cataract.

\section{The Role of microRNAs in Cataract}

\subsection{The Function of microRNAs}

MicroRNAs (miRNAs) are type of highly conserved and small, single-stranded non-coding RNAs about 20 - 22 nucleotides, that regulate the expression of target genes at post-transcriptional level. Primary miRNAs (pri-miRNAs) processed sequentially via two ribonucleases Drosha and Dicer, which belongs the RNase III family, and transcribed from miRNA genes. Mature miRNA is packed into the RNA induced silencing complex (RISC) which can directs the complex to target mRNAs, giving rise to translational repression and target mRNA degradation, by binding to the 3'untranslated region (3'UTR) of mRNA in complementary sequences [14]. MiRNAs play an important regulatory role in physiological and pathological processes, which is widely found in animals, plants and viruses. Mounting studies have shown that miRNA dysregulation plays key roles in a plethora of diseases, including cataract. Certain well-known miRNAs (such as miR-31, miR-21, and miR-224-5p) have identified as master regulators of cell proliferation, differentiation, apoptosis, cell growth, stress response and autophagy [15] [16] [17]. Ample evidence has indicated that miRNA dysregulation 
impact the procession of cataract. In particular, miRNAs are involved in the regulation of function of lens epithelial cell [18].

\subsection{The Expression and Function of miRNA in the Cataract}

Current studies indicated miRNAs have identified as a group of regulators affect the cataract formation. The dysregulation of miRNAs has discovered in a diverse range of the lens, retina and other ocular tissues, and has related with poor prognosis in a wealth of clinical cases. Most studies compared differential miRNA expression in cataract human lenses between cataract patients and normal subjects [11] [19] [20] [21] (Table 1).

The expression of miRNAs was upregulated or downregulated to induce the epithelial-to-mesenchymal transition (EMT), apoptosis, autophagy, proliferation of LECs via different signaling pathways [11] [18] [25]. Here, we summarize several relatively clear mechanisms of microRNA. For instance, miR-26a/b suppressed LEC-EMT by directly targeting Jagged-1 and controlling Jagged-1/Notch signaling pathway [11]. MiR-204-5p regulates lens epithelial cell-EMT via targeting SMAD4 during in human PCO [24]. MiRNA-181a regulates the expression of c-Met, slug, and cox-2 thereby inhibits the migration, proliferation, and EMT of lens epithelial cells [18]. Those downregulated kind of miRNA regulate the LEC-EMT through different ways, resulting in PCO. In ARC, miRNA-34a was upregulated and acted on Notch2, Bcl-2 and SIRT1 and causes apoptosis of HLECs [20] [25]. In DC, miRNA-30a was downregulated and regulated the EMT and autophagy by different pathways [22] [23].

Taken together, miRNAs could repression of those targeted protein via combine the 3'untranslated region (3'UTR) of mRNA and regulation of the different function of lens epithelium cells, and then participate in the occurrence and development of cataract (Figure 1).

Table 1. The expression and the role of miRNAs in the lens.

\begin{tabular}{|c|c|c|c|c|c|}
\hline miRNA & Expression & Target & $\begin{array}{l}\text { Cataract } \\
\text { type }\end{array}$ & Function & reference \\
\hline $\mathrm{miR}-26 \mathrm{a} / \mathrm{b}$ & down & Jagged-1/Notch & $\begin{array}{l}\text { ASC and } \\
\text { PCO }\end{array}$ & $\begin{array}{c}\text { suppress LEC } \\
\text { proliferation, } \\
\text { migration and EMT }\end{array}$ & {$[11]$} \\
\hline miR-30a & down & BECN-1 & DC & $\begin{array}{l}\text { regulation of autophagy } \\
\text { and apoptosis in LECs }\end{array}$ & {$[22]$} \\
\hline $\operatorname{miR}-30 a$ & down & SNAI1 & DC & inhibit EMT & {$[23]$} \\
\hline miRNA-204-5p & down & TGF $\beta /$ SMAD 4 & PCO & inhibit EMT & {$[24]$} \\
\hline miR-181a & down & $\begin{array}{l}\text { c-Met, slug, } \\
\text { COX-2 }\end{array}$ & $\mathrm{PCO}$ & $\begin{array}{c}\text { suppress the migration, } \\
\text { proliferation, } \\
\text { and EMT of LECs }\end{array}$ & {$[18]$} \\
\hline $\operatorname{miR}-34 a$ & up & Notch2 & $\mathrm{ARC}$ & promote LECs apoptosis & {$[25]$} \\
\hline miR-34a & up & Bcl-2, SIRT-1 & ARC & promote LECs apoptosis & {$[20]$} \\
\hline
\end{tabular}




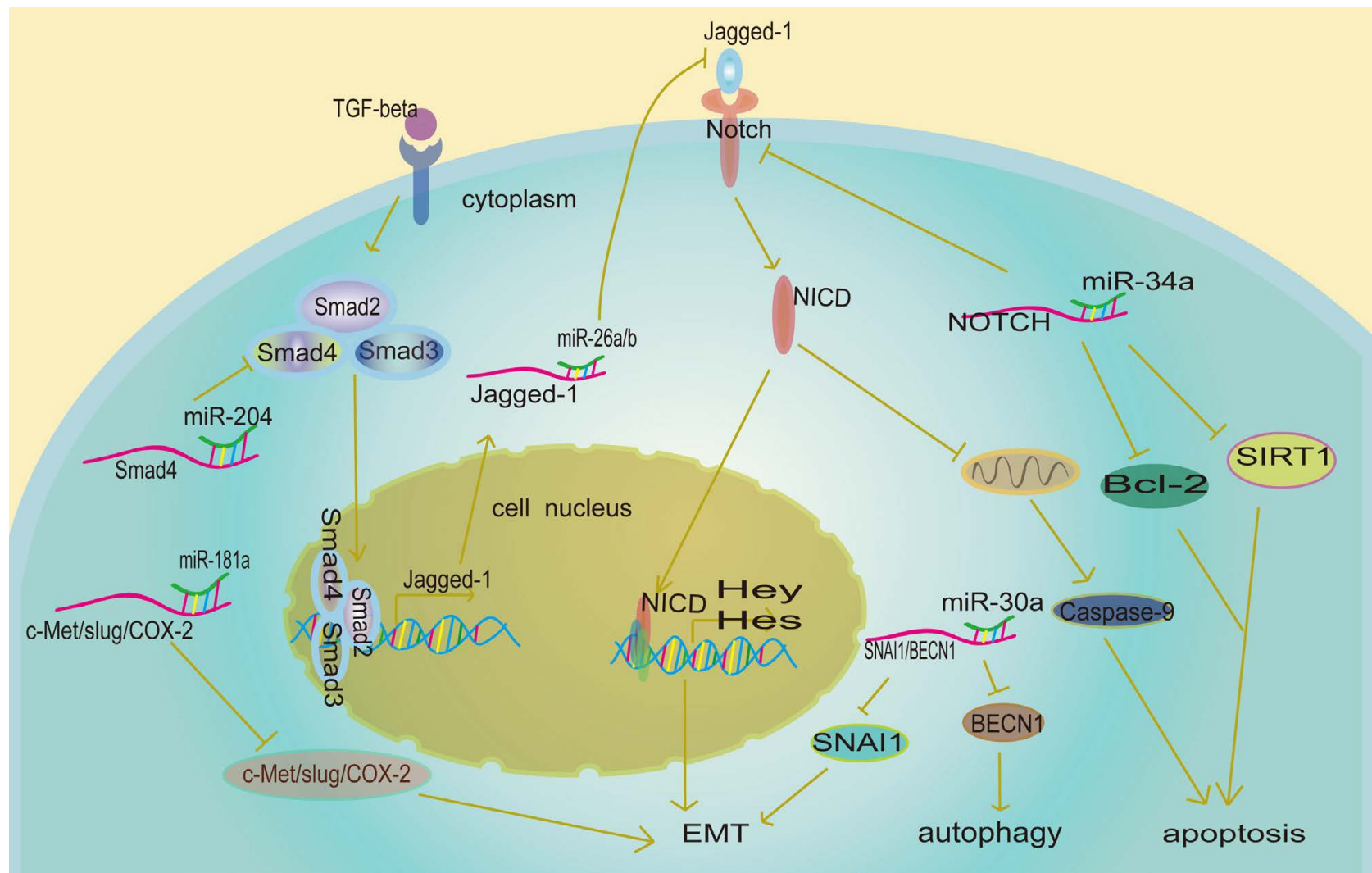

Figure 1. The role of microRNAs in cataract. MiRNAs reduce the expression of related proteins by binding to the mRNA to control the rate of translation or RNA degradation and result in EMT, autophagy, and apoptosis of HLECs.

\section{The Role of IncRNAs in Cataract}

\subsection{The Structure and Function of IncRNAs}

Long non-coding RNAs (LncRNAs) are bigger than 200 nucleotide-long RNA molecules, which lack or have limited protein-coding potential [26]. Most of IncRNAs are RNA polymerase II, whose transcription has polyA tail and 5'cap, mainly concentrated in the nucleus, which exhibit the level of evolutionary protection or expression is lower than that of mRNAs. LncRNAs regulate various aspects of gene expression via interacting with DNA, RNA or protein [27], and involved in the transcription, post-transcription of gene expression and epigenetic regulation [28]. LncRNAs play a vital role in cell differentiation regulation and many other life activities, as well as many diseases. LncRNAs are involved in regulating the ageing process, a large number of lncRNA have found to be involved in cell cycle, such as senescence proliferation and differentiation, as well as the regulation of important senescence related signaling pathways [29] [30], thereby contributing to the development of age-related diseases such as cataract.

\subsection{The Expression and Function of lncRNAs in the Cataract}

Emerging studies reveal that the dysregulation of $\operatorname{lncRNAs}$ are impact the development and progression of cataract [31] [32] [33] [34] [35]. Compared with transparent lenses, KCNQ1OT1, TUG1, and H19 has identified to have signifi- 
cantly increased expression in age-related cataract [36] [37] [38]. The significant differential expression of lncRNAs in age-related cataract human lenses indicates that lncRNAs might play a crucial role in cataract formation (Table 2).

Current researches have indicated lncRNAs exert multifaceted roles in various biological processes and disorders of cataract. Cytoplasmic lncRNAs regulate expression at the post-transcriptional level by "sponging" miRNA or interacting with RNA-binding proteins. Jin et al. demonstrated that miR-214 has a potential binding site for both KCNQ1OT1 and caspase-1 3'-UTR, KCNQ1OT1 acted as endogenous miRNA "sponge" to degrade the transcription of target genes and bind miR-214 to suppressed the miR-214 expression, consequently the HLECs impact pyroptosis, which is a type of programmed cell death by caspase- 1 mediated via inflammatory cytokines [12]. LncRNA MIAT acts as a competing endogenous RNA (ceRNA) to sponge miR-150-5p and accelerates the proliferation and migration of HLECs [34]. LncH19 was up-regulated in ARC and act as ceRNA via H19/miR-29a/TDG pathway, which may be a promising target for the treatment [36]. Another study discovered that H19/miR-675 axis may affected CRYAA expression involved in the pathogenesis of nuclear ARC [33]. CRYAA encodes the predominant structural protein participate in refractive properties and the maintenance of lens clarity. The imbalanced expression of alpha-crystallin could accumulate damage and cripple its protective effect to the lens. Therefore, H19 could be a useful marker in ARC. LncPLCD3-OT1 may act as a ceRNA to regulate the expression of PLCD3 by combining with mi-224-5p

Table 2. The expression and the role of lncRNAs in the lens.

\begin{tabular}{|c|c|c|c|c|c|}
\hline LncRNA & Target gene & $\begin{array}{l}\text { Major methods } \\
\text { and subject }\end{array}$ & Role or function & $\begin{array}{c}\text { Cataract } \\
\text { classification }\end{array}$ & reference \\
\hline KCNQ10T1 $\uparrow$ & SMAD4 & $\begin{array}{l}\text { TGF- } \beta 2 \text { induced } \\
\text { SRA01/02 in vitro }\end{array}$ & HLECs proliferation and EMT & ASC and PCO & [31] \\
\hline KCNQ10T1 $\uparrow$ & MiR-214/caspase-1 & $\begin{array}{l}\text { induced SRA01/04 } \\
\text { cell in vitro }\end{array}$ & Promote HLECs Pyroptosis, & ARC & [12] \\
\hline FEZF1-AS1 $\uparrow$ & FEZF1 & $\begin{array}{l}\text { induced SRA01/04 } \\
\text { cell in vitro }\end{array}$ & Promote HLECs proliferation & $\mathrm{PCO}$ & [39] \\
\hline MIAT $\uparrow$ & MiRNA-150-5p/Akt & SRA01/04 cell in vitro & $\begin{array}{c}\text { Increase HLECs proliferation } \\
\text { and viability; decrease } \\
\text { HLECs dead or dying }\end{array}$ & $\mathrm{PCO}$ & [34] \\
\hline $\mathrm{H} 19 \uparrow$ & miRNA-675-5p & HECs in vitro & $\begin{array}{c}\text { Increase HLECs proliferation } \\
\text { and migration, decrease } \\
\text { HLECs dead or dying }\end{array}$ & Nuclear cataract & [33] \\
\hline $\mathrm{H} 19 \uparrow$ & miR-29 & HECs in vitro & $\begin{array}{l}\text { Increase HLECs proliferation and } \\
\text { migration, decrease HLECs apoptosis }\end{array}$ & ARC & [36] \\
\hline TUG1 & MiRNA-214 & HECs in vitro & Promote HLECs apoptosis, & cataract & [37] \\
\hline MALAT1 $\uparrow$ & P38 & HECs in vitro & Promote HLECs apoptosis & Diabetic cataract & [32] \\
\hline PLCD3-OT1 $\downarrow$ & MiR-224-5P & $\begin{array}{l}\text { induced SRA01/04 } \\
\text { cell in vitro }\end{array}$ & Inhibit HLECs apoptosis & ARC & [38] \\
\hline
\end{tabular}


and also plays a protective role in ARC [38]. Highly expressed TUG1 promotes lens epithelial cell apoptosis via sponging miR-421 [37]. LncRNAs also shown to regulate gene expression by interacting with a diverse range of proteins. As an example, the elevated KCNQ1OT1 positively regulated SMAD4 expression to promote LEC proliferation and EMT in PCO [31]. LncFEZF1-AS1 promoted the proliferation and migration via upregulating FEZF1 expression in PCO [39]. Upregulated MALAT1 can activate $\mathrm{p} 38$, a component of mitogen-activated protein kinases (MAPKs) pathway, resulting in HLECs apoptosis in DC. Collectively, aberrant expression of lncRNAs contribute to cataract via different regulatory mechanisms and signaling pathways (Figure 2).

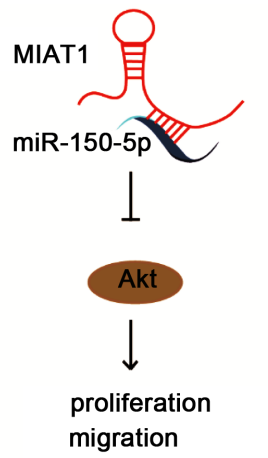

migration

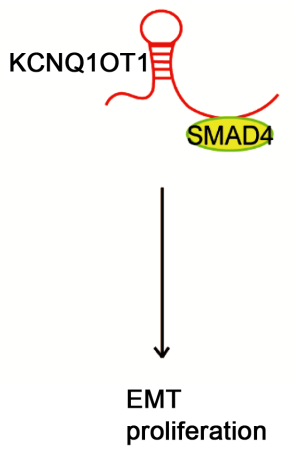

(a)

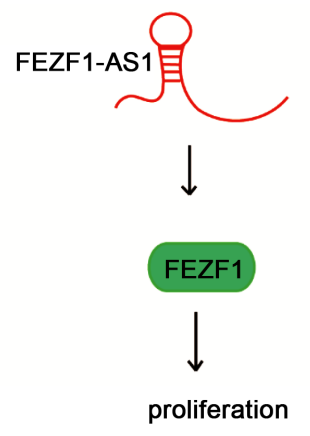

proliferation

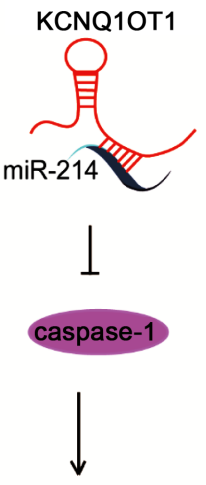

pyroptosis
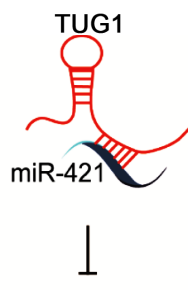

caspase-3

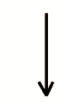

apoptosis
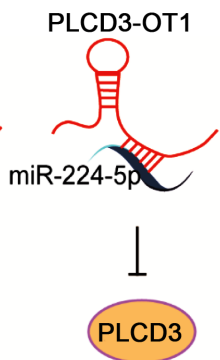<smiles>C[CH]C</smiles>

apoptosis
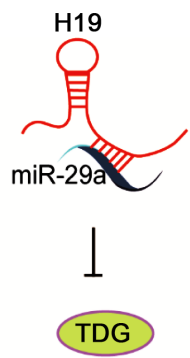

$\perp$

apoptosis

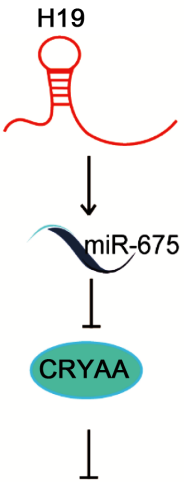

apoptosis

(b)

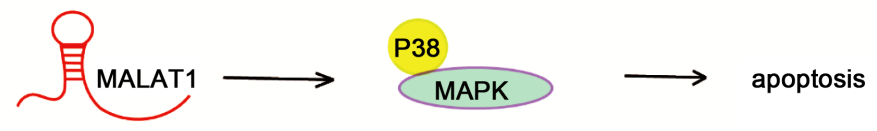

(c)

Figure 2. The role of lncRNAs in the lens. LncRNAs regulates the proliferation an EMT contribute to PCO via different pathways (a); LncRNAs regulates the pyroptosis and apoptosis contribute to age-related cataract via different pathways (b); lncRNAs regulatory mechanism in DC (c). (a) LncRNAs in ARC; (b) LncRNAs in PCO; (c) LncRNA in DC. 


\section{The Role of circRNAs in Cataract}

\subsection{The Structure and Function of circRNAs}

Circular RNAs (circRNAs) are a novel class of non-coding RNAs generated from back splicing and the covalently closed loop structure linking the $3^{\prime}$ and $5^{\prime}$ ends. CircRNAs are commonly classified into three types: exonic circRNA, exon-intronic circRNA, and circular intronic RNA [40]. The pre-mRNA was changed from a chain structure to a ring structure, and then the introns in the ring were further cut to form circRNA which has broadly studied in recent years also can serve as useful markers in many cancers [41]. Although circRNA can't achieve biological function by encoding functional protein and have a low expression level, it revealed its work by combining with miRNA as molecule sponges [42]. The miRNA sponge effect of circRNA is only the unique function of some circRNAs, rather than the general characteristics of circRNAs. Some circRNAs molecules can also bind to RNA binding protein as protein sponges [43].

\subsection{CircRNAs in the Cataract}

Circular RNAs stably expressed in a variety of biological cells, and have tissue specificity and other characteristics. Circular RNAs participate in a wide range of developmental and physiological processes, including cell apoptosis, proliferation and differentiation. Accumulating evidence have proved their crucial regulation in respective biological processes and ocular diseases. Recently, the studies on circRNAs have shown that many circRNAs play roles as microRNA sponges, RNA-binding protein sponges, transcription regulators [44]. These roles give circRNA a great potential in biological applications. CircKMT2E acted as a sponge molecule of miR-204-5p and involved in the pathogenesis of DC [45]. Liu et al. reported that CircHIPK3 are the potential regulators to regulate HLECs function in cataractogenesis through miR-193a-mediated CRYAA expression [13]. CircHIPK3 acted as a molecular sponge to adsorb miRNA-193a to inhibit the production of crystalline proteins-CRYAA. CRYAA is a protein that keeps the crystal clearly. CircHIPK3 could regulate HLECs function provide a novel insight into ARC (Figure 3).

\section{Conclusions and Future Perspectives}

In conclusion, this review mainly summarized and analyzed the physiological and pathological effects of these non-coding RNAs in the lens. The expression of non-coding RNAs modulated the diverse proteins, RNAs and signaling pathways to impair the proliferation, migration, apoptosis, pyroptosis, autophagy and abnormal EMT of HLECs. ARC is commonly caused by apoptosis, pyroptosis, autophagy of the lens epithelial cell or unbalanced expression of crystalline proteins. However, PCO is commonly caused by proliferation, metastasis, and EMT of the lens epithelial cell. In DC, non-coding RNAs may be the etiology and inducing factors to trigger the cataract, resulting in its earlier occurrence 


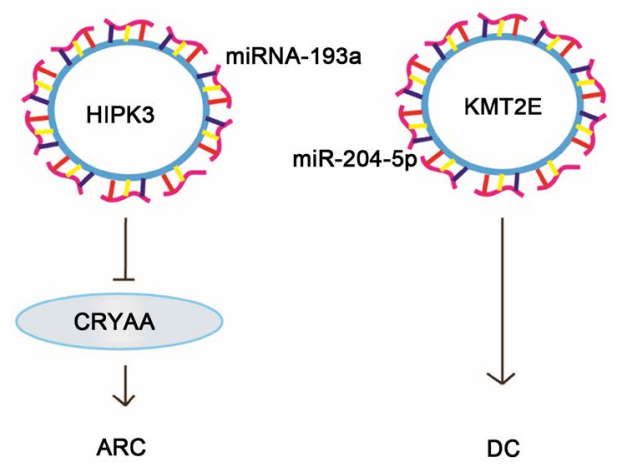

Figure 3. The mechanism of circular RNA in cataract. Circular RNA HIPK3 regulates human lens epithelial cells apoptosis by targeting the miR-193a/CRYAA axis. CircKMT2E acted as a sponge molecule of miR-204-5p and involved in the pathogenesis of DC.

and faster progress. With a growing number of ncRNAs being identified and characterized in human diseases, the emerging researches in the pre-clinical setting have shown the promise of ncRNAs as diagnostic and prognostic biomarkers. However, targeting ncRNAs in ophthalmology is still in its infancy, and improved techniques and approaches are expected in this field. In future efforts, focus should be put on a comprehensive molecular mechanism of ncRNA networks to decipher the precise roles of ncRNAs in cataract.

\section{Acknowledgements}

We would like to express our gratitude to English teacher Ze-Juan Li, from Yangtze University, for his help in writing this review and inspiring us. We would like to thank our family for giving us support. And we would like to thank our classmate Wen-Qi Cai and Jun-Ting Cheng for helping in using AI to draw all the figures.

\section{Conflicts of Interest}

The authors declare no conflicts of interest.

\section{References}

[1] Song, P., et al. (2018) The National and Subnational Prevalence of Cataract and Cataract Blindness in China: A Systematic Review and Meta-Analysis. Journal of Global Health, 8, Article ID: 010804.

[2] Hamada, N. and Sato, T. (2016) Cataractogenesis Following High-LET Radiation Exposure. Mutation Research, 770, 262-291. https://doi.org/10.1016/j.mrrev.2016.08.005

[3] Matsuda, S., et al. (2017) Aphanothece Sacrum (Sur.) Okada Prevents Cataractogenesis in Type 1 Diabetic Mice. Journal of Nutritional Science and Vitaminology (Tokyo), 63, 263-268.

[4] Shiels, A. and Hejtmancik, J.F. (2017) Mutations and Mechanisms in Congenital and Age-Related Cataracts. Experimental Eye Research, 156, 95-102. https://doi.org/10.1016/j.exer.2016.06.011

[5] Sun, X. and Wang, S. (2015) Pay Attention to the Corneal Epithelial Cell Dysfunc- 
tion after Cataract Surgery. Chinese Journal of Ophthalmology, 51, 161-162.

[6] Bouazza, M., et al. (2016) Long-Term Results of Phacoemulsification in Pseudoexfoliation Syndrome. Journal Français d Ophtalmologie, 39, 364-369.

https://doi.org/10.1016/j.jfo.2015.08.015

[7] Savastano, A., et al. (2016) Choroidal Effusion and Suprachoroidal Hemorrhage during Phacoemulsification: Intraoperative Management to Prevent Expulsive Hemorrhage. European Journal of Ophthalmology, 26, 338-341. https://doi.org/10.5301/ejo.5000707

[8] Zhao, Y., Yang, K., Li, J., Huang, Y. and Zhu, S. (2017) Comparison of Hydrophobic and Hydrophilic Intraocular Lens in Preventing Posterior Capsule Opacification after Cataract Surgery: An Updated Meta-Analysis. Medicine, 96, e8301. https://doi.org/10.1097/MD.0000000000008301

[9] Slack, F.J. and Chinnaiyan, A.M. (2019) The Role of Non-Coding RNAs in Oncology. Cell, 179, 1033-1055. https://doi.org/10.1016/j.cell.2019.10.017

[10] Wei, J.W., Huang, K., Yang, C. and Kang, C.-S. (2017) Non-Coding RNAs as Regulators in Epigenetics (Review). Oncology Reports, 37, 3-9. https://doi.org/10.3892/or.2016.5236

[11] Chen, X., et al. (2017) MicroRNA-26a and -26b Inhibit Lens Fibrosis and Cataract by Negatively Regulating Jagged-1/Notch Signaling Pathway. Cell Death \& Differentiation, 24, 1431-1442. https://doi.org/10.1038/cdd.2016.152

[12] Jin, X., Jin, H., Shi, Y., Guo, Y. and Zhang, H. (2017) Long Non-Coding RNA KCNQ1OT1 Promotes Cataractogenesis via miR-214 and Activation of the Caspase-1 Pathway. Cellular Physiology and Biochemistry, 42, 295-305. https://doi.org/10.1159/000477330

[13] Liu, X., et al. (2018) Circular RNA HIPK3 Regulates Human Lens Epithelial Cells Proliferation and Apoptosis by Targeting the miR-193a/CRYAA Axis. Biochemical and Biophysical Research Communications, 503, 2277-2285. https://doi.org/10.1016/j.bbrc.2018.06.149

[14] Catalanotto, C., Cogoni, C. and Zardo, G. (2016) MicroRNA in Control of Gene Expression: An Overview of Nuclear Functions. International Journal of Molecular Sciences, 17, 1712. https://doi.org/10.3390/ijms17101712

[15] Cheng, Y., et al. (2018) MiRNA-224-5p Inhibits Autophagy in Breast Cancer Cells via Targeting Smad4. Biochemical and Biophysical Research Communications, 506, 793-798. https://doi.org/10.1016/j.bbrc.2018.10.150

[16] Hu, X., et al. (2018) miRNA-21 Inhibition Inhibits Osteosarcoma Cell Proliferation by Targeting PTEN and Regulating the TGF-Betal Signaling Pathway. Oncology Letters, 16, 4337-4342. https://doi.org/10.3892/ol.2018.9177

[17] Luo, C., et al. (2018) miRNA-31 over-Expression Improve Synovial Cells Apoptosis Induced by RA. Bratislava Medical Journal, 119, 355-360. https://doi.org/10.4149/BLL 2018066

[18] Dong, N., Tang, X. and Xu, B. (2015) miRNA-181a Inhibits the Proliferation, Migration, and Epithelial-Mesenchymal Transition of Lens Epithelial Cells. Investigative Ophthalmology \& Visual Science, 56, 993-1001.

https://doi.org/10.1167/iovs.14-15860

[19] Tanaka, Y., et al. (2014) Profiles of Extracellular miRNAs in the Aqueous Humor of Glaucoma Patients Assessed with a Microarray System. Scientific Reports, 4, 5089. https://doi.org/10.1038/srep05089

[20] Li, Q.L., et al. (2016) MicroRNA-34a Promoting Apoptosis of Human Lens Epi- 
thelial Cells through down-Regulation of B-Cell Lymphoma-2 and Silent Information Regulator. International Journal of Ophthalmology, 9, 1555-1560.

[21] Lu, B., et al. (2018) miR-24-p53 Pathway Evoked by Oxidative Stress Promotes Lens Epithelial Cell Apoptosis in Age-Related Cataracts. Molecular Medicine Reports, 17, 5021-5028. https://doi.org/10.3892/mmr.2018.8492

[22] Zhang, L., Cheng, R. and Huang, Y. (2017) MiR-30a Inhibits BECN1-Mediated Autophagy in Diabetic Cataract. Oncotarget, 8, 77360-77368. https://doi.org/10.18632/oncotarget.20483

[23] Zhang, L., et al. (2017) MicroRNA-30a Regulation of Epithelial-Mesenchymal Transition in Diabetic Cataracts through Targeting SNAI1. Scientific Reports, 7, 1117. https://doi.org/10.1038/s41598-017-01320-3

[24] Wang, Y., et al. (2013) MicroRNA-204-5p Regulates Epithelial-to-Mesenchymal Transition during Human Posterior Capsule Opacification by Targeting SMAD4. Investigative Ophthalmology \& Visual Science, 54, 323-32. https://doi.org/10.1167/iovs.12-10904

[25] Fan, F., Zhuang, J.H., Zhou, P., Liu, X. and Lu, Y. (2017) MicroRNA-34a Promotes Mitochondrial Dysfunction-Induced Apoptosis in Human Lens Epithelial Cells by Targeting Notch2. Oncotarget, 8, 110209-110220. https://doi.org/10.18632/oncotarget.22597

[26] Ransohoff, J.D., Wei, Y. and Khavari, P.A. (2018) The Functions and Unique Features of Long Intergenic Non-Coding RNA. Nature Reviews Molecular Cell Biology, 19, 143-157. https://doi.org/10.1038/nrm.2017.104

[27] Guttman, M. and Rinn, J.L. (2012) Modular Regulatory Principles of Large Non-Coding RNAs. Nature, 482, 339-346. https://doi.org/10.1038/nature10887

[28] Roberts, T.C., Morris, K.V. and Weinberg, M.S. (2014) Perspectives on the Mechanism of Transcriptional Regulation by Long Non-Coding RNAs. Epigenetics, 9, 13-20. https://doi.org/10.4161/epi.26700

[29] Jiang, R., et al. (2017) The Long Noncoding RNA lnc-EGFR Stimulates T-Regulatory Cells Differentiation Thus Promoting Hepatocellular Carcinoma Immune Evasion. Nature Communications, 8, Article No. 15129. https://doi.org/10.1038/ncomms15129

[30] Zhang, E., et al. (2018) A Novel Long Noncoding RNA HOXC-AS3 Mediates Tumorigenesis of Gastric Cancer by Binding to YBX1. Genome Biology, 19, 154. https://doi.org/10.1186/s13059-018-1523-0

[31] Chen, B., Ma, J., Li, C.W. and Wang, Y. (2018) Long Noncoding RNA KCNQ1OT1 Promotes Proliferation and Epithelialmesenchymal Transition by Regulation of SMAD4 Expression in Lens Epithelial Cells. Molecular Medicine Reports, 18, 16-24. https://doi.org/10.3892/mmr.2018.8987

[32] Gong, W., Zhu, G., Li, J. and Yang, X. (2018) LncRNA MALAT1 Promotes the Apoptosis and Oxidative Stress of Human Lens Epithelial Cells via p38MAPK Pathway in Diabetic Cataract. Diabetes Research and Clinical Practice, 144, 314-321. https://doi.org/10.1016/j.diabres.2018.06.020

[33] Liu, X., et al. (2018) Long Non-Coding RNA H19 Regulates Human Lens Epithelial Cells Function. Cellular Physiology and Biochemistry, 50, 246-260.

https://doi.org/10.1159/000494003

[34] Shen, Y., et al. (2016) Role of Long Non-Coding RNA MIAT in Proliferation, Apoptosis and Migration of Lens Epithelial Cells: A Clinical and in Vitro Study. Journal of Cellular and Molecular Medicine, 20, 537-548. https://doi.org/10.1111/jcmm.12755 
[35] Zhang, B., Chen, Y., Qiu, M.Y. and Ding, Z. (2017) Long Noncoding RNA Expression Profile in HLE B-3 Cells during TGF-Beta2-Induced Epithelial-Mesenchymal Transition. BMC Ophthalmology, 17, 69. ttps://doi.org/10.1186/s12886-017-0461-Z

[36] Cheng, T., et al. (2019) lncRNA H19 Contributes to Oxidative Damage Repair in the Early Age-Related Cataract by Regulating miR-29a/TDG Axis. Journal of Cellular and Molecular Medicine, 23, 6131-6139. https://doi.org/10.1111/jcmm.14489

[37] Li, G., et al. (2017) TUG1 Promotes Lens Epithelial Cell Apoptosis by Regulating miR-421/Caspase-3 Axis in Age-Related Cataract. Experimental Cell Research, 356, 20-27. https://doi.org/10.1016/j.yexcr.2017.04.002

[38] Xiang, J., et al. (2019) LncRNA PLCD3-OT1 Functions as a CeRNA to Prevent Age-Related Cataract by Sponging miR-224-5p and Regulating PLCD3 Expression. Investigative Ophthalmology \& Visual Science, 60, 4670-4680.

https://doi.org/10.1167/iovs.19-27211

[39] Wang, Y., et al. (2019) LncRNA FEZF1-AS1 Promotes TGF-Beta2-Mediated Proliferation and Migration in Human Lens Epithelial Cells SRA01/04. Journal of Ophthalmology, 2019, Article ID: 4736203. https://doi.org/10.1155/2019/4736203

[40] Vicens, Q. and Westhof, E. (2014) Biogenesis of Circular RNAs. Cell, 159, 13-14. https://doi.org/10.1016/J.cell.2014.09.005

[41] Jeck, W.R., et al. (2013) Circular RNAs Are Abundant, Conserved, and Associated with ALU Repeats. RNA, 19, 141-157. https://doi.org/10.1261/rna.035667.112

[42] Chen, L.L. (2016) The Biogenesis and Emerging Roles of Circular RNAs. Nature Reviews Molecular Cell Biology, 17, 205-211. https://doi.org/10.1038/nrm.2015.32

[43] Zang, J., Lu, D. and Xu, A. (2018) The Interaction of circRNAs and RNA Binding Proteins: An Important Part of circRNA Maintenance and Function. Journal of Neuroscience Research, 98, 87-97. https://doi.org/10.1002/jnr.24356

[44] Li, X., Yang, L. and Chen, L.L. (2018) The Biogenesis, Functions, and Challenges of Circular RNAs. Molecular Cell, 71, 428-442. https://doi.org/10.1016/j.molcel.2018.06.034

[45] Fan, C., et al. (2019) Circular RNA Circ KMT2E Is up-Regulated in Diabetic Cataract Lenses and Is Associated with miR-204-5p Sponge Function. Gene, 710, 170-177. https://doi.org/10.1016/j.gene.2019.05.054 